\title{
In-situ Heating TEM Study of Phase Change Material
}

\author{
Jia $\mathrm{Li}^{1 *}$, Liang Hong ${ }^{1}$ and Haifeng Wang ${ }^{1}$ \\ 1. Western Digital Corporation®, 44100 Osgood Road, Fremont, CA. \\ *Jia Li: Jia.Li@wdc.com
}

Ge2Sb2Te5 (GST) is one of the phase change materials (PCM) currently used for non-volatile memory (NVM) storage based on its structural phase transitions. At elevated temperatures, phase change takes place from amorphous phase to crystalline phase ${ }^{[1]}$. The crystallization transition temperature is dependent upon temperature ramp rate and has a wide range from $140^{\circ} \mathrm{C}$ to $170^{\circ} \mathrm{C}$ [2] . In the past, many studies have been carried out to investigate physical property change between amorphous and crystalline phases of GST film, such as density and resistivity ${ }^{[3]}$. However, the precise crystallization transition temperature is not easily attained due to many difficulties including heat dosage and thermal gradient control, nanoscale effect and time to equilibrium.

In this paper, we will cover methodology on how to precisely calibrate the phase transition temperature of nanoscale GST film using in-situ heating TEM. Real time phase transition from amorphous GST film to crystalline film can be directly detected using TEM imaging and electron diffraction analysis.

Our experimental set up includes FEI Tecnai TF20 TEM and Protochips Aduro 300 holder. The Protochips heating holder gives us precise temperature and ramping control that enables in-situ heating of the nanoscale GST film in TEM. Selected area electron diffraction (SAED) is employed to precisely detect the phase transition from amorphous phase to FCC crystalline phase with increasing film temperatures. Unlike conventional use of thermal chips, 20nm GST is directly sputtered onto the chips. The electrical leads are masked during the film deposition so that the conductive path stays intact after deposition and chip's temperature calibration remains unchanged.

To characterize GST crystallization transition temperature and investigate the dependency of this temperature upon heat dosage, three types of temperature ramp rates combined with soak times are used with three thermal chips during in-situ heating. For Chip 1, temperature is first ramped instantaneously to $120^{\circ} \mathrm{C}$, then increased by $5^{\circ} \mathrm{C}$ step followed by 6 seconds soak time every step. For Chip 2, temperature is linearly fast ramped to $120^{\circ} \mathrm{C}$ in 15 seconds, whereas for chip 3 , it is slowly ramped to $120^{\circ} \mathrm{C}$ in 600 seconds to mimic conventional hot plate ramping process. From $120^{\circ} \mathrm{C}$ to $165^{\circ} \mathrm{C}$, ramp rate is $5^{\circ} \mathrm{C} / 15$ seconds, followed by 60 seconds sock time at every step for both Chip 2 and Chip 3. More details of the heating conditions can be found in Figure 1. Bright Field TEM images and diffraction patterns are collected at every $5^{\circ} \mathrm{C}$ soak step to monitor the GST phase and the transition point.

The GST films in both Chips 2 and 3 exhibit consistent amorphous to crystalline phase change at $150^{\circ} \mathrm{C}$, whereas the crystallization occurs at $160^{\circ} \mathrm{C}$ in Chip 1 . These findings are consistent with previous studies ${ }^{[2]}$, and confirm that GST crystallization transition temperature decreases with increasing heat dosage. However, below $120^{\circ} \mathrm{C}$, larger heat dosage from slower ramp rate does not affect phase transition point. Such in-situ heating TEM methodology can be utilized to study the properties of a broad range of nanoscale PCM memory devices. 


\section{References:}

[1] Lombardo, S., Rimini, E., Grimaldi, M. G., \& Privitera, S., Amorphous-fcc transition in Ge2Sb2Te5. Microelectronic Engineering, 87(3), (2010), 294-300.

[2] Friedrich, V. Weidenhof, W. Njoroge, P. Franz, and M. Wuttig, Structural transformations of Ge2Sb2Te5 films studied by electrical resistance measurements, Journal of Applied Physics, 87(9), (2000), 4131-4134.

[3] Njoroge, W. K., Wöltgens, H. W., \& Wuttig, M., Density changes upon crystallization of Ge $2 \mathrm{Sb}$ 2.04 Te 4.74 films. Journal of Vacuum Science \& Technology A: Vacuum, Surfaces, and Films, 20(1), (2002), 230-233.

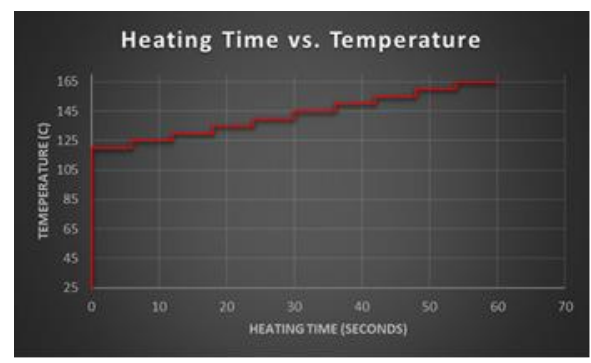

Chip 1

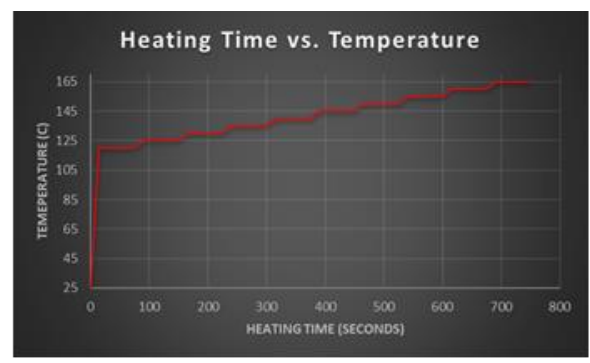

Chip 2

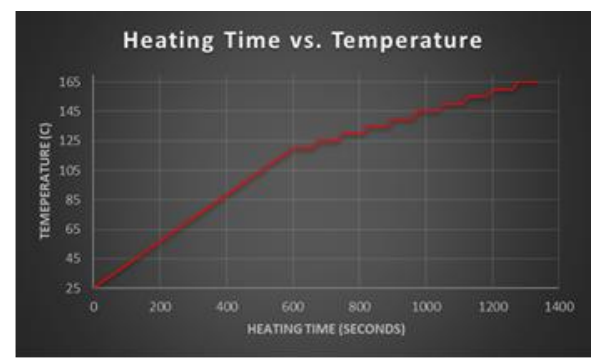

Chip 3

Figure 1. In-Situ TEM heating experimental conditions
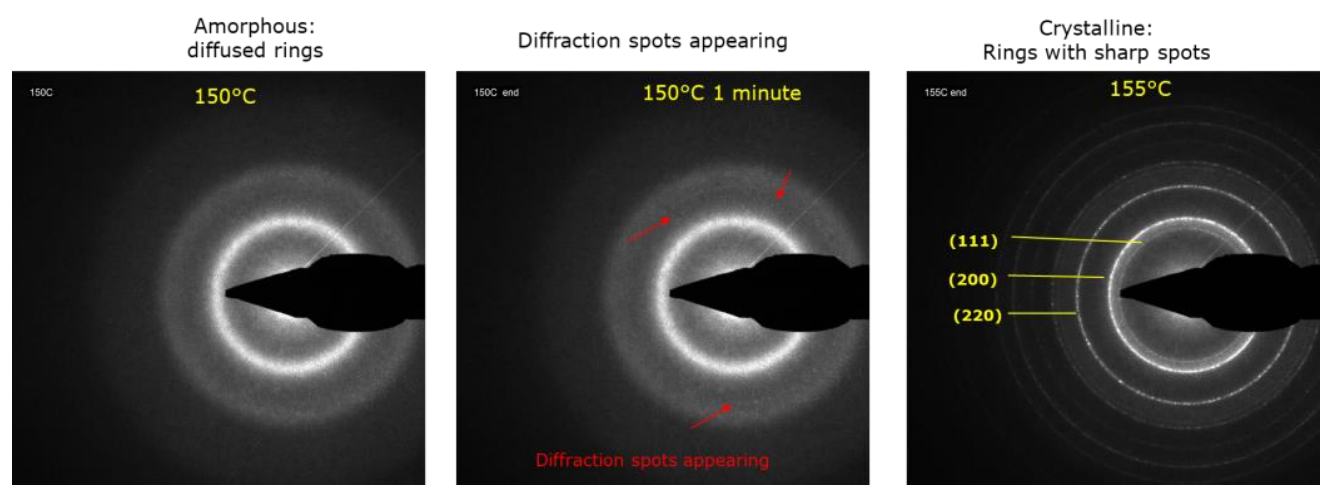

Figure 2. GST phase transition at $150^{\circ} \mathrm{C}$ Amorphous
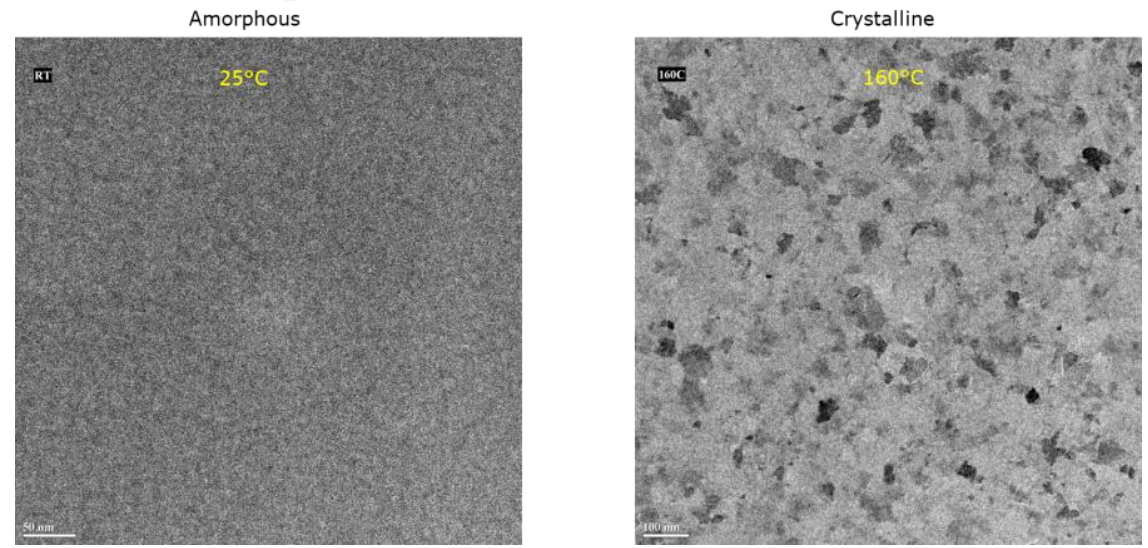

Figure 3. GST phase transition from amorphous to crystalline 